\title{
RETRACTED ARTICLE: Diagnostic and prognostic potentials of KLF6 and HER3 expression alterations in cutaneous malignant melanoma
}

\author{
Ali Ebrahimi ${ }^{1}$ - Seyed Mohamad Hossein Tabatabaei Nodushan ${ }^{2}$ - Alireza Mousavian ${ }^{3}$. \\ Aram Mokarizadeh ${ }^{4} \cdot$ Mehdi Abbasi $^{5} \cdot$ Emad Yahaghi $^{6} \cdot$ Seyed Mohammad Rasaei $^{7}$
}

Published online: 16 October 2015

(C) International Society of Oncology and BioMarkers (ISOBM) 2016

\section{RETRACTED ARTICLE: Tumor Biol.}

DOI 10.1007/s13277-015-4236-y

This article has been retracted at the request of the Editorin-Chief, the International Society of Oncology and BioMarkers (ISOBM) and the Publisher per the Committee on Publication Ethics guidelines. The article shows evidence of irregularities in authorship and in the materials and methods during the submission process, and there is strong reason to believe that the peer review process was compromised.

Electronic supplementary material The online version of this article (doi:10.1007/s13277-015-4236-y.) contains supplementary material, which is available to authorized users.

Seyed Mohammad Rasaei

rasaei66sm@yahoo.com

1 Students' Research Committee, Baqiyatallah University of Medical Sciences, Tehran, Iran

2 Faculty of Medicine, Shahid Beheshti University of Medical Sciences and Health Services, Tehran, Iran

3 Department of Orthopedics Surgery, Faculty of Medicine, Mashhad University of Medical Sciences, Mashhad, Iran

4 Cellular and Molecular Research Center and Department of Immunology, Kurdistan University of Medical Sciences, Sanandaj, Iran

5 School of Medicine, Shahed University, Tehran, Iran

6 Department of Molecular Biology, Baqiyatallah University of Medical Sciences, Tehran, Iran

7 Arak University of Medical Sciences, Arak, Iran
As such the validity of the content of this article cannot be verified.

The online version of this article contains the full text of the retracted article as electronic supplementary material. 